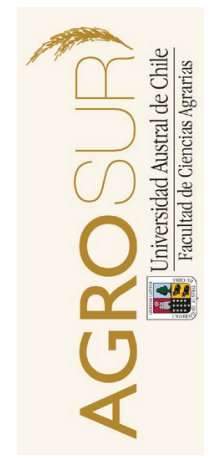

\title{
Efecto de la frecuencia de defoliación en el rendimiento y composición nutricional de Pennisetum clandestinum Hochst.ex Chiov
}

\author{
Effect of defoliation frequency on yield and nutritional composition \\ of Pennisetum clandestinum Hochst.ex Chiov \\ Fonseca, C. ${ }^{a}$ Balocchi, 0. ${ }^{a *}$, Keim, J.P. ${ }^{a}$, Rodríguez, C. ${ }^{b}$ \\ ${ }^{a}$ Facultad de Ciencias Agrarias, Universidad Austral de Chile \\ ${ }^{b}$ Facultad de Ciencias Agropecuarias, Universidad Pedagógica y Tecnológica de Colombia, Tunja, \\ Colombia. Calle 13 No.27-60 Duitama, Colombia.
}

\begin{abstract}
A B S T R A C T
In Colombia at 2,560 m.a.s.l. the effect of three defoliation frequencies $\left(3,4.5\right.$ and 6 leaves tiller $\left.{ }^{-1}\right)$ was evaluated, during four months (August - November), on growth and nutritional quality of Kikuyu (Pennisetum clandestinum Hochst.ex Chiov). Nine plots of $6 \times 4$ m were established, which were distributed in a complete randomized block repeated measures design. Data were subjected to analysis of variance. A significant interaction between defoliation frequency and the month of utilization on total herbage mass yield and crude protein concentration was observed. The highest values for both variables were obtained at 6 leaves tiller ${ }^{-1}$ in October (herbage mass) and OctoberNovember (crude protein). Defoliation frequency did not modify yield components (proportion of blades, sheath, stems and dead material). Neutral Detergent Fiber and Acid Detergent Fiber were not affected by defoliation frequency. In conclusion, grazing of kikuyu swards, under similar environmental conditions of this study should be performed at stage of 4.5 to 6 leaves tiller ${ }^{-1}$ with a residual sward height of $5 \mathrm{~cm}$.
\end{abstract}

\section{RESUMEN}

En Colombia a 2.560 m.s.n.m, se evaluó por cuatro meses (Agosto - Noviembre) el efecto de tres frecuencias de defoliación (basadas en la aparición de 3; 4,5 y 6 hojas macollo-1) sobre el crecimiento y calidad nutritiva del kikuyu (Pennisetum clandestinum Hochst.ex Chiov). Se establecieron nueve parcelas de 6 x 4 m, en un diseño experimental de bloques completos al azar con medidas repetidas en el tiempo. Los datos fueron sometidos a análisis de varianza. Se observó interacción significativa entre la frecuencia de defoliación y los meses de utilización sobre la fitomasa total y sobre el contenido de proteína cruda. Los mayores valores para ambas variables se obtuvieron con 6 hojas macollo-1 en el mes de octubre (fitomasa) y 6 hojas macollo-1 en octubre - noviembre (proteína cruda). La frecuencia de defoliación no modificó los componentes del rendimiento (proporción de láminas, vainas, tallos y material muerto). Los valores de fibra detergente neutra y fibra detergente ácida fueron similares en todos los tratamientos de defoliación. En conclusión, el pastoreo de esta especie, en condiciones ambientales similares a las de éste estudio, deberá realizarse en el estado de 4,5 a 6 hojas macollo-1 respetándose la altura de residuo de $5 \mathrm{~cm}$.

Palabras clave: componentes de rendimiento, fitomasa, pradera tropical, kikuyu.

\section{INTRODUCCIÓN}

La frecuencia de defoliación es una variable que ha mostrado tener un significativo efecto en la producción y calidad nutricional de una pradera (Barbosa et al., 2011). Esto ha sido ampliamente demostrado en especies de clima templado, sin embargo, en especies de clima tropical existe menos información disponible. Pennisetum clandestinum Hochst. ex Chiov, es una gramínea tropical C4 constituida por 36 cromosomas (Crush y Rowarth, 2007; Ludlow, 1985; Mears, 1970) que acumula almidones en las células del mesófilo (McDonald et al., 2002) y soporta altas concentraciones de $\mathrm{CO}_{2}$ (Dood et al., 2010). Presenta un alto rendimiento de fitomasa (Ludlow, 1985), lo que le permite soportar una alta carga animal (Colman y Kaiser, 1974), contiene una baja calidad nutritiva por los altos niveles de fibra y baja digestibilidad (Lowe et al., 2010), con desbalance en mi- 
nerales en comparación con otras gramíneas perennes templadas, pero con una excelente respuesta a la fertilización y riego (Bernal, 2008; Read y Fulkerson, 2003). Resiste el pisoteo de los animales y tolera las sequías debido a su profundo sistema radical (Marais, 2001).

El $P$. clandestinum es utilizado para pastoreo en los sistemas lecheros de Colombia. Los sistemas de producción basados en praderas deben tener una base de sustentación para conocer el punto óptimo de utilización como fuente primordial de la rentabilidad del sistema (García et al., 2014). Estudios realizados por Reeves et al. (1996) en Australia sugieren que para lograrlo, se debe pastorear el $P$. clandestinum cuando éste tenga 4,5 hojas macollo ${ }^{-1}$, momento en que se logra una calidad nutricional adecuada a los requerimiento de vacas en lactancia. Al pasar ese estado, los parámetros nutricionales decrecen debido al incremento del tallo y la acumulación de material muerto (Chapman y Lemaire, 1993).

La cantidad de fitomasa recomendada para pastorear una pradera de kikuyu es de $2.600 \mathrm{~kg}$ de materia seca (MS) ha-1, cuando sólo se cosechan entre 1.100 a $1.500 \mathrm{~kg} \mathrm{MS} \mathrm{ha}^{-1}$ (Fariña et al., 2011; García et al., 2014). Por esta razón, la intensidad y frecuencia de pastoreo serán parte fundamental para mantener una adecuada relación hoja:tallo cuando se deja un residuo de pastoreo entre los 5-6 cm de altura (Chacón y Stobbs, 1976; Sinclair et al., 2007; Fulkerson et al., 2010).

En Colombia, no existen publicaciones sobre el efecto de frecuencias de pastoreo en base número de hojas sobre la calidad nutricional del forraje, volviéndose un tema relevante para los sistemas eficientes de producción. Al ser un país de localización tropical sin estaciones marcadas, sería importante conocer el comportamiento agronómico de sus praderas debido a que la mayoría de las investigaciones reportadas en P. clandestinum se encuentran en lugares geográficos diferentes como Australia, Brasil y Sudáfrica con alturas sobre el nivel del mar diferentes a las que se abordará en este estudio (Botha et al., 2008a; Botha et al., 2008b; Vander Colf et al., 2013).

El objetivo de esta investigación fue determinar el efecto de la frecuencia de defoliación en P. clandestinum sobre el rendimiento y calidad nutritiva de la pradera.

\section{MATERIALES Y MÉTODOS}

\section{Sitio}

El estudio se realizó en un periodo de 4 meses (Agosto - Noviembre 2014), en un campo agropecuario de lechería especializada en el Trópico Alto Colombiano en el municipio de Santa Rosa de Viterbo (Boyacá) a 2.560 msnm, localizado a $5^{\circ} 49^{\prime} 42^{\prime \prime}$ de latitud norte y $1^{\circ} 2^{\prime} 48^{\prime \prime}$ longitud en relación con el meridiano de Bogotá y $73^{\circ}$ $03^{\prime}$ longitud al oeste de Greenwich con una precipitación promedio anual de $849 \mathrm{~mm}$ en forma de lluvia (Gobernación de Boyacá, 2014; IDEAM, 2015; IGAC, 1995).
Para homogenizar el sitio, el área experimental fue cortada al inicio del estudio a una altura residual de $5 \mathrm{~cm}$, el 29 de julio de 2014 (Sinclair et al., 2007; Fulkerson et al., 2010). Las parcelas del ensayo fueron establecidas en praderas de kikuyu de una antigüedad superior a 30 años, sobre un suelo de origen aluvial, catalogado como pesado serie Río Chicamocha (Bernal, 2014; Municipio de Duitama, 2014). El pH del suelo se encuentra dentro de los valores adecuados para su crecimiento como lo reportan Awad et al. (1976) siendo éste de 5,6. Los valores de minerales del suelo son de $\mathrm{N}=43,2 ; \mathrm{P}=45,1$ (método Bray II); $\mathrm{K}=321 ; \mathrm{Mg}=475$; $\mathrm{Ca}=3.997$ ppm de suelo.

$\mathrm{Al}$ inicio del experimento se aplicó una fertilización de mantención correspondiente a $185 \mathrm{~kg} \mathrm{ha}^{-1}$ de una mezcla comercial que contenía $31-8-8 \%$ de N-P-K, respectivamente. Durante el ensayo, se realizaron otras dos aplicaciones más mensuales de esta misma mezcla, con un total de $172 \mathrm{~kg} \mathrm{ha}^{-1}$ de $\mathrm{N}$ y $44 \mathrm{~kg} \mathrm{ha}^{-1}$ de $\mathrm{P}$ y K para todo el estudio.

Los promedios de temperatura tanto ambiental, del suelo y los grados acumulados día fueron obtenidos de los registros llevados en el campo por medio de instrumentos de medición como termómetro ambiental de mínimas - máximas y digital de suelo $(10 \mathrm{~cm}$ de profundidad). Los grados día acumulados fueron determinados considerando $8^{\circ} \mathrm{C}$ como temperatura base (Muscolo, 2013) (Cuadro 1).

La cantidad de agua regada fue programada con un requerimiento mensual de $150 \mathrm{~mm}$, calculado con la precipitación semanal y el déficit correspondiente cubierto por riego de aspersión en las horas de la tarde (Cuadro 2).

\section{Condiciones climáticas}

Las condiciones de temperatura presentadas durante el periodo del experimento, mostraron un promedio de $15,6^{\circ} \mathrm{C}$ en el ambiente, de $16,3^{\circ} \mathrm{C}$ en el suelo y de $23,2^{\circ} \mathrm{C}$ acumulados mes (base de $8^{\circ} \mathrm{C}$ ) (Cuadro 1 ).

Los valores de agua suministrados a la pradera fueron determinados por las horas de riego por aspersión y el volumen de precipitación mensual. El promedio diario entregado fue de $9,31 \mathrm{~mm}$ con una evapotranspiración de 3,7 $\mathrm{mm}$ tomada por metodología bandeja tipo A (Ortega y Acevedo, 1999; Zhai, 2010), ofreciendo un total mensual de $167 \mathrm{~mm}$ de agua (Cuadro 2).

\section{Variables evaluadas}

\section{Rendimiento de fitomasa, altura de la pradera y componentes del rendimiento}

Cuando cada tratamiento alcanzó el número de hojas correspondiente de 3, 4,5 o 6 hojas macollo-1 ${ }^{-1}$ se midió la altura no disturbada con una regla de medi- 
Cuadro 1. Valores de temperaturas promedio mensual $\left({ }^{\circ} \mathrm{C}\right)$ durante el estudio.

Table 1. Average monthly temperature $\left({ }^{\circ} \mathrm{C}\right)$ during the study.

\begin{tabular}{lcccc}
\hline & Ambiente & Suelo & & Grados acumulado mes \\
\cline { 2 - 3 } & \multicolumn{2}{c}{$\left({ }^{\circ} \mathrm{C}\right.$ promedio $)$} & & (base $\left.8^{\circ} \mathrm{C}\right)$ \\
\hline Agosto & 14,6 & 16,9 & & 204 \\
Septiembre & 16,1 & 15,4 & & 243 \\
Octubre & 15,9 & 16,6 & 245 \\
Noviembre & 15,9 & 16,3 & \\
Temporada & 15,6 & 16,3 & & 237 \\
\hline
\end{tabular}

Cuadro 2. Cantidad de precipitaciones y riego aplicado durante el estudio.

Table 2. Amount of rainfall and irrigation applied per month.

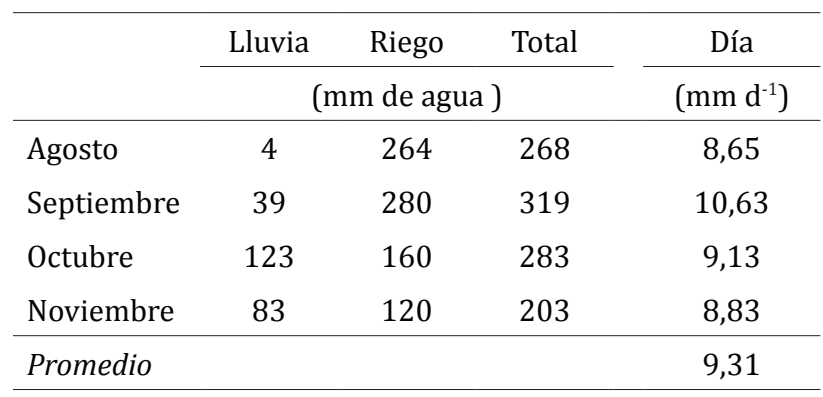

ción de $30 \mathrm{~cm}$, con diez observaciones por parcela. Se procedió a eliminar el borde y posteriormente a cosechar las parcelas con una cortadora de césped dejando una altura de residuo de $5 \mathrm{~cm}$. Se pesó en fresco todo el material cosechado de cada parcela. Se tomaron tres submuestras cada una de $100 \mathrm{~g}$, una para determinar calidad nutritiva, otra para determinar concentración de materia seca por medio del horno de ventilación forzada a $60^{\circ} \mathrm{C}$ por 48 horas y otra para obtener los porcentajes de cada uno de los componentes del rendimiento (lámina, vaina, tallo y material muerto). Posteriormente, se tomaron 3 muestras de $50 \times 50 \mathrm{~cm}$ del material por debajo de los $5 \mathrm{~cm}$ de altura para determinar la biomasa acumulada de residuo ha- ${ }^{-1}$ y a su vez, los componentes del rendimiento bajo la altura de corte.

\section{Calidad nutricional}

La calidad nutricional fue determinada en una de las submuestras tomada a $5 \mathrm{~cm}$ de altura de residuo antes de ser cosechada la parcela y a la misma hora en cada uno de los tratamientos (medio día). Los análisis fueron realizados en el Laboratorio de Nutrición Animal de la Facultad de Ciencias Agropecuarias de la Universidad Pedagógica y Tecnológica de Colombia sede Tunja, Boyacá. La concentración de proteína cruda (PC) en la pradera fue obtenida por el método de micro-Kjeldahl según Bateman (1970) obteniendo la cantidad de nitrógeno total y multiplicando por la constante de 6,25. La concentración de fibra detergente neutra (FDN) y fibra detergente ácida (FDA) fueron determinadas por el método de Van Soest et al. (1991), según lo reportado por AOAC (1996).

\section{Diseño experimental y análisis estadístico}

Para el análisis de los datos todos los resultados fueron agrupados por mes. Los tratamientos se distribuyeron en 9 parcelas de $6 \times 4 \mathrm{~m}^{2}$ ordenadas en tres bloques, para evaluar tres frecuencias de defoliación de 3, 4,5 y 6 hojas macollo-1 ${ }^{-1}$ estableciéndose un diseño en bloques completos al azar con medidas repetidas en el tiempo. Los datos fueron sometidos a un análisis de medidas repetidas utilizando el procedimiento PROC MIXED de SAS® (9.4).

Adicionalmente se incluyó un efecto aleatorio para evaluar el componente de la varianza entre bloques. Las diferencias entre los tratamientos se determinaron utilizando la opción PDIFF con Tukey ajustado en SAS®. Los patrones de covarianza no estructurada evaluados fueron, Simetría Compuesta y Autorregresivo de primer orden. El patrón de covarianza final fue elegido mediante los criterios de información bayesianos (AIC y BIC, respectivamente), considerando de entre ellos el que convergiera y obtuviese el valor más bajo.

\section{RESULTADOS}

\section{Rendimiento de fitomasa}

$\mathrm{Al}$ analizar los promedios de tasa crecimiento y fitomasa total de MS ha- ${ }^{-1}$ durante el periodo de estudio para cada una de las frecuencias de defoliación de $3,4,5$ y 6 hojas macollo-1 ${ }^{-1}$ se observó que no existieron diferencias estadísticamente significativas $(p>0,05)$ entre ellas. Sin embargo, considerando la ausencia de diferencias significativas, se observa que numéricamente la frecuencia de 6 hojas fue la que presentó la mayor tasa de crecimiento y rendimiento total de materia seca en comparación con las frecuencias de defoliación de 3 y 4,5 hojas respectivamente (Cuadro 3 ).

Para las variables tasa crecimiento y producción de fitomasa existió una interacción significativa $(p<0,05)$ entre las frecuencias de defoliación y los meses de estudio. La interacción se produce únicamente en el mes de noviembre encontrándose que la mayor tasa de crecimiento y producción de fitomasa se observó con la menor frecuencia de defoliación ( 6 hojas maco$1 \mathrm{lo}^{-1}$ ) comparado con la mayor frecuencia de defoliación (3 hojas macollo-1) pero no presento diferencia 
Cuadro 3. Tasa de crecimiento y rendimiento de fitomasa total para las frecuencias de defoliación 3; 4,5 y 6 hojas macollo-1.

Table 3. Growth rate and total herbage mass for defoliation frequencies of 3; 4.5 and 6 leaves tiller ${ }^{-1}$.

\begin{tabular}{|c|c|c|}
\hline & Tasa crecimiento diaria & Fitomasa Total \\
\hline & $\left(\mathrm{kg} \mathrm{MS} \mathrm{d}^{-1}\right)$ & $\left(\mathrm{kg} \mathrm{ha}^{-1}\right)$ \\
\hline \multicolumn{3}{|c|}{ Frecuencia de defoliación } \\
\hline 3 hojas & 75,4 & 6.787 \\
\hline 4,5 hojas & 77,4 & 6.966 \\
\hline 6 hojas & 95,3 & 8.576 \\
\hline valor $P$ & 0,092 & 0,092 \\
\hline e.e. & 7,2 & 650 \\
\hline
\end{tabular}

significativa con la frecuencia de 4,5 hojas macollo ${ }^{-1}$ (Cuadro 4).

\section{Fitomasa residual y altura de la pradera}

La fitomasa residual presentó interacción significativa entre frecuencias de defoliación y meses de estudio $(\mathrm{p}<0,05)$, observándose que en noviembre la frecuencia de 3 hojas macollo ${ }^{-1}$ es mayor en $\mathrm{kg} \mathrm{MS} \mathrm{ha}^{-1}$ que las frecuencias de 4,5 y 6 hojas macollo ${ }^{-1}$ respectivamente. La fitomasa residual obtenida mostró diferencias altamente significativas entre las frecuencias de defoliación y los meses del estudio $(\mathrm{p}<0,01)$ encontrándose que la frecuencia de 6 hojas macollo-1 presentó la menor cantidad de kg MS ha-1 en comparación con las otras dos

Cuadro 4. Rendimiento de fitomasa y altura de la pradera previo al corte en las diferentes frecuencias de defoliación durante los meses de estudio.

Table 4. Herbage production and sward height as affected by defoliation frequencies, month of the year and their interaction.

\begin{tabular}{|c|c|c|c|c|}
\hline & Tasa de crecimiento & Fitomasa total & Fitomasa residual & Altura \\
\hline & $\left(\mathrm{kg} \mathrm{MS} \mathrm{d}^{-1}\right)$ & $\left(\mathrm{kg} \mathrm{ha}^{-1}\right)$ & $\left(\mathrm{kg} \mathrm{ha}^{-1}\right)$ & $(\mathrm{cm})$ \\
\hline \multicolumn{5}{|c|}{ Frecuencia de defoliacion } \\
\hline 3 hojas & 75,4 & 2.262 & 6.614 & 10,9 \\
\hline 4,5 hojas & 77,4 & 2.322 & 6.206 & 14,0 \\
\hline 6 hojas & 95,3 & 2.859 & 5.119 & 17,5 \\
\hline valor $P$ & 0,092 & 0,092 & 0,0026 & $<0,001$ \\
\hline e.e. & 7,2 & 217 & 251 & 0,6 \\
\hline \multicolumn{5}{|l|}{ Meses } \\
\hline Septiembre & 71,4 & 2.142 & 4.975 & 13,2 \\
\hline Octubre & 91,8 & 2.753 & 6.710 & 13,7 \\
\hline Noviembre & 84,9 & 2.547 & 6.253 & 15,6 \\
\hline valor $P$ & $<0,001$ & $<0,001$ & $<0,001$ & $<0,001$ \\
\hline e.e. & 3,9 & 117 & 318 & 0,5 \\
\hline \multicolumn{5}{|l|}{ Interaccion } \\
\hline \multicolumn{5}{|l|}{ Septiembre } \\
\hline 3 hojas & 76,8 & 2.303 & 5.244 & 10,9 \\
\hline 4,5 hojas & 70,7 & 2.121 & 5.335 & 14,0 \\
\hline 6 hojas & 66,7 & 2.002 & 4.345 & 14,7 \\
\hline \multicolumn{5}{|l|}{ Octubre } \\
\hline 3 hojas & 83,8 & 2.513 & 6.707 & 10,7 \\
\hline 4,5 hojas & 79,2 & 2.376 & 7.300 & 13,3 \\
\hline 6 hojas & 112,4 & 3.370 & 6.122 & 17,0 \\
\hline \multicolumn{5}{|l|}{ noviembre } \\
\hline 3 hojas & 65,7 & 1.970 & 7.889 & 11,0 \\
\hline 4,5 hojas & 82,3 & 2.468 & 5.982 & 14,7 \\
\hline 6 hojas & 106,8 & 3.203 & 4.889 & 21,0 \\
\hline valor $P$ & 0,001 & 0,001 & 0,031 & 0,002 \\
\hline e.e. & 9,09 & 272,7 & 515,26 & 0,92 \\
\hline
\end{tabular}


frecuencias 3 y 4,5 hojas macollo ${ }^{-1}$ respectivamente. Respecto a los meses, se presentó menor cantidad de kg MS ha-1 en el mes de septiembre comparado con los meses de octubre y noviembre (Cuadro 4).

La altura obtenida al corte mostró una interacción significativa entre las frecuencias de defoliación y los meses de estudio $(p<0,01)$, encontrándose en el mes de septiembre que las alturas fueron similares entre las frecuencia de 4,5 y 6 hojas macollo-1 ${ }^{-1}$ pero mostraron diferencia significativa con la mayor frecuencia de defoliación (3 hojas macollo ${ }^{-1}$ ). En octubre las alturas de las frecuencia de 3 y 4,5 hojas macollo ${ }^{-1}$ fueron similares pero hubo diferencia altamente significativa entre la de 6 hojas con la de 3 hojas macollo ${ }^{-1}$ y en noviembre se encontraron diferencias altamente significativas entre las frecuencias de defoliación de 3, 4,5 con la de 6 hojas macollo ${ }^{-1}$ y diferencia significativa entre la frecuencia de 3 y 4,5 hojas macollo-1 (Cuadro 4).

\section{Componentes del rendimiento}

Para fitomasa cosechada sobre $5 \mathrm{~cm}$ de altura, no se encontró interacción significativa entre frecuencias de defoliación y meses de estudio. No se encontró diferencias significativas para los componentes del rendimiento entre las diferentes defoliaciones, pero si se encontraron diferencias altamente significativas entre los meses de estudio para los componentes de láminas, tallos y material muerto $(\mathrm{p}<0,01)$. Dentro del componente de láminas, se observó un menor porcentaje en el mes de noviembre en relación a los meses de septiembre y octubre, mientras que el porcentaje de los tallos fue mayor en noviembre en relación a los meses de septiembre y octubre siendo septiembre el de menor valor. La proporción de material muerto fue mayor en el mes de septiembre en relación a los meses de octubre y noviembre (Cuadro 5).

Cuadro 5. Componentes del rendimiento en las diferentes frecuencias de defoliación durante los meses de estudio.

Table 5. Yield components as affected by defoliation frequency, month of the year and their interaction.

\begin{tabular}{|c|c|c|c|c|c|c|c|c|}
\hline & \multicolumn{4}{|c|}{$\%$ Material Cosechado } & \multicolumn{4}{|c|}{ \% Material Residual } \\
\hline & Laminas & Vainas & Tallos & Material muerto & Laminas & Vainas & Tallos & Material muerto \\
\hline \multicolumn{9}{|c|}{ Frecuencia de defoliación } \\
\hline 3 hojas & 56,9 & 17,6 & 16,8 & 9,0 & 23,9 & 16,1 & 26,3 & 33,6 \\
\hline 4,5 hojas & 59,0 & 16,6 & 15,7 & 8,8 & 13,1 & 12,9 & 35,7 & 38,3 \\
\hline 6 hojas & 57,5 & 16,3 & 16,6 & 9,7 & 12,9 & 11,5 & 36,9 & 28,7 \\
\hline valor $P$ & 0,653 & 0,438 & 0,668 & 0,617 & 0,070 & 0,19 & 0,003 & 0,316 \\
\hline e.e. & 2,2 & 1,0 & 1,2 & 0,9 & 4,3 & 2,1 & 1,5 & 3,4 \\
\hline \multicolumn{9}{|l|}{ Meses } \\
\hline Septiembre & 60,2 & 16,0 & 10,9 & 13,22 & 15,9 & 11,7 & 27,8 & 44,7 \\
\hline Octubre & 60,3 & 15,4 & 17,0 & 7,26 & 18,9 & 12,2 & 32,6 & 36,3 \\
\hline Noviembre & 52,9 & 18,9 & 21,2 & 6,93 & 15,3 & 18,9 & 38,5 & 29,6 \\
\hline valor $P$ & 0,004 & 0,069 & $<0,001$ & 0,004 & 0,597 & 0,056 & 0,011 & $<0,001$ \\
\hline e.e. & 2,0 & 1,5 & 1,3 & 1,7 & 3,7 & 2,0 & 2,9 & 2,7 \\
\hline \multicolumn{9}{|l|}{ Interacción } \\
\hline \multicolumn{9}{|l|}{ Septiembre } \\
\hline 3 hojas & 60,6 & 16,4 & 11,3 & 12,6 & 20,9 & 14,3 & 20,6 & 44,3 \\
\hline 4,5 hojas & 61,8 & 15,3 & 10,4 & 12,6 & 16,3 & 11,6 & 28,8 & 43,3 \\
\hline 6 hojas & 58,2 & 16,4 & 10,9 & 14,5 & 10,4 & 9,2 & 34,0 & 46,4 \\
\hline \multicolumn{9}{|l|}{ Octubre } \\
\hline 3 hojas & 61,0 & 16,1 & 15,4 & 7,5 & 34,4 & 12,5 & 24,9 & 28,2 \\
\hline 4,5 hojas & 59,0 & 15,1 & 17,5 & 8,4 & 10,1 & 12,3 & 36,9 & 40,7 \\
\hline 6 hojas & 60,8 & 15,2 & 18,2 & 5,9 & 12,2 & 11,9 & 36,0 & 39,9 \\
\hline \multicolumn{9}{|l|}{ Noviembre } \\
\hline 3 hojas & 49,1 & 20,4 & 23,7 & 6,8 & 16,5 & 21,6 & 33,5 & 28,3 \\
\hline 4,5 hojas & 56,1 & 19,3 & 19,3 & 5,3 & 13,1 & 14,8 & 41,3 & 30,8 \\
\hline 6 hojas & 53,4 & 17,2 & 20,7 & 8,7 & 16,3 & 13,5 & 40,6 & 29,7 \\
\hline valor $P$ & 0,370 & 0,885 & 0,356 & 0,678 & 0,124 & 0,578 & 0,834 & 0,331 \\
\hline e.e. & 3,5 & 2,3 & 2,2 & 2,5 & 6,8 & 3,5 & 4,4 & 5,12 \\
\hline
\end{tabular}


Para la fitomasa residual, por debajo de $5 \mathrm{~cm}$ de altura de corte, no se encontró interacción significativa entre frecuencias de defoliación y meses de estudio. Se presentaron diferencias altamente significativas en la proporción de tallos en las frecuencias de defoliación $(p<0,01)$ observándose que la frecuencia de 3 hojas macollo ${ }^{-1}$ mostró una menor proporción de tallos en relación a las frecuencias de 4,5 y 6 hojas macollo-1 (Cuadro 5).

Analizando los componentes de rendimiento del material residual en cada uno de los meses de estudio, se encontró diferencias altamente significativas en la proporción de tallos y material muerto $(\mathrm{p}<0,01)$, observándose un mayor porcentaje de tallos en el mes de noviembre en comparación con el mes de septiembre. El porcentaje de tallos en el mes de octubre no presentó diferencias significativas con ninguno de los otros dos meses $(32,62 \% \pm 2,93$ e.e.). En cuanto al material muerto, el mes de septiembre presentó el mayor porcentaje observándose diferencias significativas con los meses de octubre y noviembre (Cuadro 5).

\section{Calidad nutritiva}

Para FDN y FDA, no se presentó interacción significativa entre frecuencias de defoliación y meses de estudio. La frecuencia de defoliación no modificó significativamente $(p>0,05)$ estas variables. Los meses de estudio modificaron la concentración de FDN $(\mathrm{p}<0,05)$ mostrándose una mayor concentración en los meses de octubre y noviembre siendo similares entre sí pero presentando una diferencia significativa con el mes de septiembre como se observa en el Cuadro 6, sin embargo la concentración de FDA no fue diferente.

Para la concentración de PC, se presentó interacción significativa entre frecuencias de defoliación y meses de estudio. Las frecuencias de defoliación modificaron significativamente la concentración de PC. En los meses de estudio, las diferencias fueron altamente significativas $(\mathrm{p}<0,01)$.

En la interacción, el mes de octubre mostró diferencias significativas entre las tres frecuencias siendo mayor la frecuencia de 6 hojas macollo ${ }^{-1}$ y en el mes de noviembre la menor concentración de PC se dio con la frecuencia de 3 hojas macollo ${ }^{-1}$ mostrándose diferencias significativas con las frecuencias de 4,5 y 6 hojas macollo $^{-1}$ las cuales fueron similares entre ellas.

Las concentraciones de PC en las frecuencias de 3 y 4,5 hojas macollo ${ }^{-1}$ y entre 4,5 y 6 hojas macollo ${ }^{-1}$ mostraron similitud entre ellas pero con diferencias significativas entre la frecuencia de 3 y 6 hojas maco${ }_{1 \mathrm{o}^{-1}}$. Respecto a los meses, se presentan diferencias significativas entre los tres meses siendo noviembre el de mayor porcentaje de PC y septiembre el de menor porcentaje (Cuadro 6).
Cuadro 6. Concentraciones de PC, FDN y FDA en las diferentes frecuencias de defoliación durante los meses de estudio.

Table 6. Chemical composition (CP, NDF and ADF) as affected by defoliation frequency, month of the year and their interaction.

\begin{tabular}{|c|c|c|c|}
\hline & PC & FDN & FDA \\
\hline & \multicolumn{3}{|c|}{$\% \mathrm{MS}$} \\
\hline \multicolumn{4}{|c|}{ Frecuencia de defoliación } \\
\hline 3 hojas & 25,3 & 54,3 & 26,5 \\
\hline 4,5 hojas & 26,8 & 54,5 & 26,8 \\
\hline 6 hojas & 27,3 & 54,5 & 27,4 \\
\hline valor $P$ & 0,028 & 0,970 & 0,636 \\
\hline e.e. & 0,55 & 0,98 & 0,92 \\
\hline \multicolumn{4}{|l|}{ Meses } \\
\hline Septiembre & 23,1 & 52,4 & 27,1 \\
\hline Octubre & 26,1 & 55,6 & 26,5 \\
\hline Noviembre & 30,2 & 55,4 & 27,1 \\
\hline valor $P$ & $<, 0001$ & 0,022 & 0,801 \\
\hline e.e. & 0,6 & 1,1 & 1,0 \\
\hline \multicolumn{4}{|l|}{ Interacción } \\
\hline \multicolumn{4}{|l|}{ Septiembre } \\
\hline 3 hojas & 23,7 & 52,0 & 26,9 \\
\hline 4,5 hojas & 22,8 & 53,1 & 27,3 \\
\hline 6 hojas & 23,0 & 52,2 & 27,1 \\
\hline \multicolumn{4}{|l|}{ Octubre } \\
\hline 3 hojas & 24,3 & 56,5 & 27,6 \\
\hline 4,5 hojas & 26,4 & 56,5 & 26,1 \\
\hline 6 hojas & 27,5 & 53,6 & 25,8 \\
\hline \multicolumn{4}{|l|}{ Noviembre } \\
\hline 3 hojas & 28,1 & 54,5 & 25,1 \\
\hline 4,5 hojas & 31,3 & 55,0 & 27,0 \\
\hline 6 hojas & 31,3 & 57,8 & 29,3 \\
\hline valor $P$ & 0,036 & 0,152 & 0,265 \\
\hline e.e. & 1,0 & 1,8 & 1,7 \\
\hline
\end{tabular}

\section{DISCUSIÓN}

En este estudio, los rendimientos de fitomasa cosechados en las diferentes frecuencias de defoliación 3, 4,5 y 6 hojas macollo ${ }^{-1}$ no presentaron diferencias estadísticas a pesar de obtenerse una mayor cantidad de producción total en el tratamiento de 6 hojas macollo $^{-1}$. Reeves et al. (1996) en Australia, consideran que el $P$. clandestinnum por ser una gramínea tropical C4 debe ser regulada por su calidad nutricional y no por su rendimiento de fitomasa total debido a las limitantes 
de consumo animal que presenta por sus valores altos de fibra, mientras que García et al. (2014) mencionan que no solo influye la alta cantidad de pared celular sino también las bajas concentraciones de carbohidratos hidrosolubles y el desbalance de minerales como los principales limitantes de esta especie. En Colombia, la mayor cantidad de materia seca se produce en época lluviosa (marzo-abril-mayo y octubre-noviembre) con un intervalo de defoliación de 30 días (Vela y Vargas, 2009), lo cual sería consistente con los resultados de este estudio en donde la mayor cantidad de fitomasa se obtuvo con las 6 hojas macollo- ${ }^{-1}$ con un intervalo de defoliación de 37 días $\pm 5,5$ (promedio \pm e.e.) en los meses de octubre y noviembre época lluviosa del año. En contraste, las menores producciones de materia seca en este estudio se obtuvieron en el mes de septiembre. IDEAM (2005) categorizó el año en dos temporadas secas (desde enero a febrero y desde julio hasta agosto) y dos lluviosas (desde finales de marzo a junio y desde octubre hasta principios de diciembre). En los meses secos de la primera temporada llueve alrededor de 7 días mientras que en los de mitad de año llueve en promedio 15 días $\mathrm{mes}^{-1}$ respectivamente. En la época lluviosa llueve alrededor de 15 a 20 días mes ${ }^{-1}$ (IDEAM, 2015).

El intervalo más corto de aparición de hoja (tiempo en días que toma una hoja para extenderse completamente) fué de 4,4 días, obtenido en un intervalo de defoliación de 24,3 días $\pm 2,6$ (promedio \pm e.e.), en el tratamiento de 4,5 hojas macollo ${ }^{-1}$, mientras que el intervalo de aparición de hoja más largo fue de 8 días encontrándose en un intervalo de defoliación de 37 días $\pm 5,5$ (promedio \pm e.e.) en el tratamiento de 6 hojas macollo ${ }^{-1}$. Los mayores intervalos de aparición de hoja se encontraron en el primer corte de las tres frecuencias de defoliación obtenidas del crecimiento de la planta a partir del mes de agosto con una temperatura de $14,6^{\circ} \mathrm{C} \pm 0,5$ (promedio \pm e.e.), resultados que corroboraron lo escrito por Herrero et al. (2000a; 2000b); Chapman y Lemaire, (1993) quienes relacionaron la tasa de aparición de hojas con la temperatura encontrándose una disminución en el tiempo a medida que ésta se incrementó.

La fitomasa residual encontrada por debajo de los $5 \mathrm{~cm}$ de corte fue mayor a medida que se incrementa la frecuencia de defoliación pero sin afectar la fitomasa total, presentándose una relación de fitomasa disponible para oferta de un $30 \% \pm 0,02$, en relación a la fitomasa total existente a ras de suelo. Briske et al. (1994) reportan que las continuas defoliaciones estimulan la producción de nuevos tallos constantemente, razón que podría ser utilizada como estrategia de protección en suelos arcillosos en épocas de lluvia.

La altura de la pradera al momento de cosecharse, presentó diferencias significativas en el mes de noviembre siendo mayor en el tratamiento de 6 hojas macollo $^{-1}$ con $17 \mathrm{~cm} \pm 0,92$ en comparación con los tra- tamientos de 3 y 4,5 hojas de 10,8 y $14 \mathrm{~cm}$ respectivamente. Dobos et al. (2009a, 2009b) relacionan altura de pastoreo con consumo animal demostrando que a $13 \mathrm{~cm}$ se presenta el mayor consumo de materia seca, siendo este valor similar a la altura reportada en este estudio con el tratamiento de 4,5 hojas macollo ${ }^{-1}$. Por otro lado, Sbrissia et al. (2003) reportan que al cosechar a $10 \mathrm{~cm}$ de altura, el aprovechamiento del forraje será de un $67 \%$ debido a la baja acumulación de tallos dentro de la pradera, confirmando así la relación directa entre hoja:tallo.

La proporción de componentes de rendimiento del material cosechado, mostró diferencias en el mes de noviembre en relación a los otros dos meses, coincidiendo con Rautenbach et al. (2008), quienes reportan que en temporada de invierno la proporción de láminas en relación a los tallos disminuye. Respecto al material muerto, las diferencias encontradas en el mes de septiembre fueron dadas por la presentación de heladas con $-4^{\circ} \mathrm{C}$ finalizando el mes de agosto, las cuales influyeron sobre el primer mes cosechado en septiembre en las diferentes frecuencias de defoliación.

Rautenbach et al. (2008), encuentran una proporción de láminas de 56-65 \% que concuerda con lo observado en este estudio $57,78 \% \pm 2,18$ (promedio \pm e.e.). Fulkerson et al. (1999), reportan una disminución en la proporción de hojas a medida que se incrementa la frecuencia de defoliación, este fenómeno no fué observado en este estudio donde no se encontró diferencia significativa entre las diferentes frecuencias de defoliación. Las proporciones de hoja (lámina y vainas) vs. tallos se encuentran en un $74,5 \%$ vs. $25,4 \% \pm 0,53$ (promedio \pm e.e.) siendo similares entre las diferentes frecuencias de defoliación, manteniendo así una baja proporción de material muerto dentro de la pradera lo que conlleva a una adecuada oferta forrajera para el consumo del animal, considerando un manejo adecuado de defoliación a la altura de $5 \mathrm{~cm}$. Fulkerson et al. (2010) demuestran que esta especie debe pastorearse entre 5 a $8 \mathrm{~cm}$ de residuo. Fulkerson et al. (1999), reportan que a mayores alturas de residuo $(12 \mathrm{~cm}) \mathrm{la}$ proporción de material muerto se incrementa, razón por la cual recomienda pastorear a 5-6 cm con 4 hojas, concordando con la altura reportada para corte en este estudio. Chapman et al. (1993), sugieren que al llevarse a cabo pastoreos severos la calidad nutricional de la pradera mejora, no obstante, es necesario buscar el punto óptimo entre elevada calidad nutricional y altas tasas de crecimiento. Con pastoreos adecuados la cantidad de tallos disminuye considerablemente como también el material muerto mejorándose la digestibilidad del forraje.

En este estudio se puede observar que la relación de los componentes del rendimiento no fue diferente en ninguno de los tratamientos dado por el manejo adecuado de la altura de corte y de la capacidad de la 
planta para captar la luz (Laca y Lemaire, 2000; Lowe, 2010), permitiendo una calidad nutritiva similar y una baja cantidad de material muerto.

Aunque Reeves et al. (1996), reportan una gran caída en la calidad nutricional después de las 4,5 hojas macollo ${ }^{-1}$, esto se debe a los cambios encontrados en la relación hoja:tallo. Los valores encontrados en este estudio no son similares, ya que la calidad nutricional referida a FDN y FDA no mostraron diferencias significativas en ninguna de las frecuencias de defoliación confirmándose por la homogeneidad de los resultados de la relación hoja:tallo. Los valores de FDN y FDA obtenidos se encuentran por debajo de los reportados por Correa et al. (2008), y Marais (2001), sin embargo, similares a los reportados por Miles et al. (2000).

Respecto a la PC, Correa et al. (2008); Reeves et al. (1996); Soto et al. (2005), y Vela y Vargas (2009), encuentran valores promedios menores a los obtenidos en este estudio.

Soto et al. (2005), al evaluar dosis de fertilización sobre la calidad nutricional de pradera de kikuyu, demuestran que ésta no influye sobre la PC, mientras que Mejía et al. (2014) reportan que a medida que se incrementa la fertilización, los valores de PC aumentan alcanzando el 20,04\% en praderas de 45 días con dosis de $50 \mathrm{~kg} \mathrm{~N} \mathrm{ha}^{-1}$. Loaiza et al. (2016) sugieren que con fertilización y pastoreos intensos la concentración de PC se incrementa hasta un 38\%. Whitney (1974) en un estudio realizado con 3 diferentes dosis de fertilización (22, 56 y $\left.168 \mathrm{~kg} \mathrm{ha}^{-1}\right)$ con intervalos de aplicación de 10 semanas evaluó rendimiento total, \% PC, mostrando que con niveles altos de fertilización $(168 \mathrm{~kg}$ ha $^{-1}$ ) los valores de PC pueden alcanzar $30 \%$ a los 30 días y disminuir a partir de los 42 días, mientras que con dosis de 22 y $56 \mathrm{~kg} \mathrm{ha}^{-1}$ los valores de PC alcanzan un 20\%. Osorio (1996) coincide con Loaiza et al. (2016), en que altos niveles de fertilización nitrogenada incrementan la PC por encima de un $25 \%$ siendo similar a los resultados obtenidos en este estudio con dosis de fertilización mensual de $44 \mathrm{de} \mathrm{N} \mathrm{kg} \mathrm{ha}^{-1}$ (Fulkerson, 2007).

Nuestros resultados indican que no habría diferencias importantes al pastorear en cualquiera de las 3 frecuencias de defoliación estudiadas. Sin embargo, debe tenerse en cuenta otros aspectos antes de recomendar este tipo de estrategias. Fulkerson et al. (1999) señalan que las vacas consumen las $2 / 3$ partes de la pradera ofrecida sobre $5 \mathrm{~cm}$ de altura equivalentes a un 70 $80 \%$ de la cobertura total, mientras que Marais (2001) menciona un consumo máximo por vacas de $1.200 \mathrm{~kg}$ MS ha-1 en cada pastoreo. García et al. (2014) sugieren que las vacas deberán cosechar entre 1.000 y $1.300 \mathrm{~kg}$ MS ha-1 en cada pastoreo y Fariña et al. (2011) recomiendan una cobertura de fitomasa de $2.600 \mathrm{~kg} \mathrm{MS} \mathrm{ha}^{-1}$ con un consumo de $1.500 \mathrm{~kg} \mathrm{MS} \mathrm{ha}^{-1}$ en cada pastoreo. Tomando en consideración las investigaciones men- cionadas, solo el tratamiento de 6 hojas macollo ${ }^{-1}$ del presente estudio cumpliría con las cantidades de cobertura de fitomasa de $2.600 \mathrm{~kg} \mathrm{MS} \mathrm{ha}^{-1} \mathrm{y}$ de consumo de $1.500 \mathrm{~kg} \mathrm{MS} \mathrm{ha}^{-1}$. Por otra parte, las cantidades de forraje ofrecidas a un animal siempre estarán limitadas por la cantidad de fibra que este contenga. Más aún en praderas como kikuyu en donde la producción está restringida por el consumo de forraje dado por las cantidades de FDN altas que esta especie contiene. Correa et al. (2008); Kolver y Muller (1998); Marais (2001) sugieren cantidades máximas de consumo de kikuyu de $13 \mathrm{~kg} \mathrm{MS}$ vaca $^{-1} \mathrm{~d}^{-1}$, dado por el 1,2, 1,5 a 1,6\% del peso vivo de FDN, mientras que Henning et al. (1995) reportan consumos de hasta $15,7 \mathrm{~kg} \mathrm{MS}$ vaca $^{-1} \mathrm{~d}^{-1}$ equivalente al 2,8\% del peso vivo. García et al. (2014) confirman que ésto podría ser incierto, ya que estudios han demostrado la habilidad de algunas vacas para consumir valores mayores al 2\% de su peso vivo en FDN dado por la digestibilidad ruminal de la materia orgánica en las diferentes especies forrajeras, mostrándose una digestibilidad por encima del 85\% en gramíneas C3 (Avena spp. y Lolium spp.) y una hasta del $59-62 \%$ en gramíneas C4 (P. clandestinnum).

Con los valores reportados en este estudio, se podría obtener consumos de $13 \mathrm{~kg}$ MS para un animal de $600 \mathrm{~kg}$ sin importar la frecuencia de defoliación dado por las similares concentraciones de FDN presentadas en las tres defoliaciones (54\%) equivalentes al 1,2\% del peso vivo (Mertens, 1987).

En el presente estudio no se analizó la digestibilidad, pero si es importante destacar la similar calidad nutricional encontrada en todos los tratamientos y la homogénea relación hoja:tallo de las diferentes defoliaciones, siendo estos parámetros fundamentales en el momento de una buena utilización de la pradera y un adecuado consumo del animal.

Aunque la hipótesis planteada en este estudio no se cumplió al no presentarse un incremento en el rendimiento de fitomasa y en la proporción de lámina con una menor calidad nutritiva al disminuir la frecuencia de defoliación en el $P$. clandestinnum, debe resaltarse que esto pudo estar influenciado por el manejo adecuado de las defoliaciones respetando la altura de residuo de 5-6 cm para obtener una buena calidad nutricional dada por la relación hoja:tallo.

\section{CONCLUSIONES}

La frecuencia de defoliación como estrategia de pastoreo en la especie $P$. clandestinum en condiciones similares a las reportadas en esta investigación bajo riego y fertilización, sugieren la utilización de esta gramínea dentro del rango de frecuencia de defoliación de 4,5 y 6 hojas macollo ${ }^{-1}$ donde se reportan la mejor calidad nutritiva dada por la homogénea relación existente de hoja:tallo a una altura de ingreso a pastoreo de 14 a 
$17,5 \mathrm{~cm}$ con una fitomasa total entre 2.350 a $2.900 \mathrm{~kg}$ MS ha-1 y una altura residual de $5 \mathrm{~cm}$.

Se sugiere para próximas investigaciones con esta especie, evaluarla bajo diferentes altitudes y temperaturas por un mayor período de tiempo comparándola bajo condiciones naturales de humedad y fertilidad.

\section{AGRADECIMIENTOS}

Agradecemos a la Hacienda Casa Vieja (Santa Rosa de Viterbo, Boyacá) por proporcionar las instalaciones y apoyo financiero al programa y al laboratorio de nutrición animal de la Facultad de Ciencias Agropecuarias de la Universidad Pedagógica y Tecnológica de Colombia (Tunja, Boyacá) por facilitar las instalaciones y equipos para el procesamiento de muestras de esta investigación.

\section{REFERENCIAS}

Awad, A.S., Edward, D.G., Milham, P.J., 1976. Effect of soil pH and phosphate on soluble aluminium and on growth and composition of kikuyu grass. Plant and Soil 45, 531-542.

AOAC, 1996. Official Methods of Analysis of AOAC International $16^{\text {th }}$ ed. Método 973.18. Gaithersburg MD, USA.

Bateman, J.V., 1970. Nutrición Animal: Manual de métodos analíticos. Ed. Herrero Hermanos, México, pp. 463-468.

Barbosa, R., Domicio, J.R., Helio, V., Carneiro Da Silva, R., Valeiria, P., Batista, E., Sbrissia, A., Sousa, B., 2011. Morphogenic and structural characteristic of guinea pastures submitted to three frequencies and two defoliation severities. Revista Brasileira de Zootecnia 40, 947-954.

Bernal, J., 2008. Pastos y Forrajes Tropicales. $5^{\text {a }}$ Ed. Manejo de Praderas. Stilos Impresoras Ltda. Bogotá, Colombia, pp. 385-420.

Bernal, E., 2014. Geografía Cultural de Boyacá. Aspectos físico-bióticos y clasificación del suelo. Recopilación datos de FAO, INCORA, CAR y IGAC. http://www.boyacacultural.com/index.php?option=com_content\&view=article\&id $=24 \&$ Itemid $=25$

Briske, D.D., Richards, J.H., 1994. Physiological responses of individual plants to grazing: current status and ecological significance. Ecological implications of livestock herbivory in the west. Society for Range Management, Denver, pp. 147-176.

Botha, P.R., Meeske, R., Snyman, H.A., 2008a. Kikuyu oversown with ryegrass and clover: dry matter, production botanical and nutritional value. African Journal of Range and Forage Science 25(3), 93-101.

Botha, P.R., Meeske, R., Snyman, H.A., 2008b. Kikuyu oversown with ryegrass and clover: grazing capacity, milk production and milk composition. African Journal of Range and Forage Science 25(3), 103-110.

Colman, R.L., Kaiser, A., 1974. The effect of stocking rate on milk production from Kikuyu grass pastures fertilized whit nitrogen. Journal of Animal Science 14, 155-160.

Correa, C., Pabón, M.L., Carulla, J.E., 2008. Valor nutricional del pasto kikuyo (Pennisetum clandestinum Hoechst Ex Chiov) para la producción de leche en Colombia: Una re- visión: I - Composición química y digestibilidad ruminal y posruminal. Livestock Research for Rural Development 20(4). http://www.lrrd.org/lrrd20/4/corra20059.htm

Chacón, E., Stobbs, T.H., 1976. Influence of progressive defoliation of a grass sward on the eating behavior of cattle. Australian Journal of Agricultural Research 27, 709-727.

Chapman, D.F., Lemaire, G., 1993. Interaction between plant physiology and pasture feeding value: a review. Crop and Pasture Science 65, 721-734.

Chapman, D.F., Lee, J.M., Waghorn, G.C., 1993. Morphogenetic and structural determinants of plant regrowth after defoliation. Proceeding Grasslands for our world. Proc. $17^{\text {th }}$ International Grasses Congress Hamilton, New Zealand Grassland Association. pp. 95-104.

Crush, J.R., Rowarth, J.S., 2007. The role of C4 grasses in New Zealand pastoral systems. New Zealand Journal of Agricultural Research 50, 115-137.

Dobos, R.C., Fulkerson, W.J., Sinclar, K., Hinch, G.N., 2009a. Grazing behaviour and pattern of intake of dairy cows grazing kikuyu (Pennisetum clandestinum) grass pastures in relation to sward height and length of grazing session. Animal Production Science 49, 233-238.

Dobos, R.C., Sinclair, K., Hinch, G.N., Fulkerson, W.J., 2009 b. Frequency distribution of sward height of kikuyu (Pennisetum clandestinum) grass pastures intensively grazed by dairy cows. Animal Production Science 49, 574-585.

Dood, M.B., Newton, P.C.D., Liefferring, M., Luo, D., 2010. The responses of three $\mathrm{C} 4$ grasses to elevated and $\mathrm{CO}_{2}$ in the field. Proceedings of the New Zealand Grassland Association 72, 61-66.

Fariña, S.R., García, S.C., Fulkerson, W.J., 2011. A complementary forage system whole-farm study: forage utilization and milk production. Animal Production Science 51(5), 460-470.

Fulkerson, B., 2007. Kikuyu grass (Penniseyum clandestinum). Future Dairy, Tech note, pp. 1-7. http://futuredairy.com. au/wp-content/uploads/2016/02/TechNoteKikuyu.pdf

Fulkerson, B., Griffiths, N., Sinclair, K., Beale, P., 2010. Milk production from Kikuyu grass based pastures. Prime Facts, Australia, pp. 2-13. http://www.jingeri.com/ wp-content/uploads/2014/11/Milk-production-fromkikuyu-grass-based-pastures.pdf

Fulkerson, W.J., Slack, K., Havilah, E., 1999. The effect of defoliation interval and height on growth and herbage quality of kikuyu grass (Pennisetum clandestinum). Tropical Grasslands 33, 138-145.

García, S.C., Islam, M.R., Clark, C.E.F., Martin, P.M., 2014. Kikuyu-based pasture for dairy production: a review. Crop and Pasture Science 65(8), 787-797.

Gobernación de Boyacá, 2014. Aspectos Geográficos de Boyacá, Colombia. http://www.boyaca.gov.co/prensa-publicaciones/mi-boyac\%C3\%A1/aspectos-geograficos

Herrero, M., Fawcett, R.H., Silveira, V., Busqué, J., Bernués, A., Dent, J.B., 2000a. Modelling the growth and utilization of kikuyu grass (Pennisetum clandestinum) under grazing. 2. Model definition and parameterization. Agricultural Systems 65, 73-97.

Herrero, M., Fawcett, R.H., Dent, J.B., 2000b. Modelling the growth and utilization of Kikuyu grass (Pennisetum clandestinum) under grazing. 2. Model validation and analysis of management practices. Agricultural Systems 
65, 99-111.

Henning, W.P.H., Barnard, H.H., Venter, J.J., 1995. Effect of grazing cycle on milk production of cows on kikuyu pastures. South African Journal of Animal Science 25(1), 7-12.

Instituto de Hidrología, Meteorología y Estudios Ambientales (IDEAM), 2015. Características climatológicas de ciudades principales y municipios turísticos de Colombia. http://institucional.ideam.gov.co/jsp/1772

Instituto Geográfico Agustín Codazzi (IGAC), 1995. Suelos de Colombia. Bogotá, Colombia, pp. 5-18.

Kolver, E.S., Muller, L.D., 1998. Performance and nutrient intake of high producing Holstein cows consuming pasture or a total mixed ration. Journal of Dairy Science 81, 1403-1411.

Laca, E., Lemaire, G., 2000. Field and Laboratory methods for grassland and animal production research. Measuring sward structure. Capítulo 5. Cabi Publishing. New York, USA, pp. 104-110.

Loaiza, P., Balocchi, O.A., Bertrand, A., 2016. Carbohydrate and crude protein fractions in perennial ryegrass as affected by defoliation frequency and nitrogen application rate. Grass and Forage Science (Published On-line doi: 10.1111/gfs.12258).

Lowe, K.F., Bowdler, T.M., Sinclair, K., Holton, T.A., Skabo, S.J., 2010. Phenotypic and genotypic variation within populations of kikuyu (Pennisetum clandestinum) in Australia. Tropical Grasslands 44, 84-94.

Ludlow, M., 1985. Photosynthesis and dry matter production in $\mathrm{C}_{3}$ and $\mathrm{C}_{4}$ pasture plants, with special emphasis on tropical $\mathrm{C}_{3}$ legumes and $\mathrm{C}_{4}$ grasses. Australian Journal of Plant Physiology 12, 557-572.

Marais, J.P., 2001. Factors affecting the nutritive value of kikuyu grass (Pennisetum clandestinum): a review. Tropical Grasslands 35, 65-84.

Mertens, D.R., 1987. Predicting intake and digestibility using mathematical models of ruminal function. Journal of Animal Science 64, 1548-1558.

McDonald, P., Edwards, R., Greenhalgh, J., Morgan, C., 2002. Nutrition animal. Gosport, England.

Mears, P.T., 1970. Kikuyu (Pennisetum clandestinum) as a pasture grass: a review. Tropical Grasslands 4, 2.

Mejía, A.C., Ochoa, R., Medina, M., 2014. Efecto de diferentes dosis de fertilizante compuesto en la calidad del pasto kikuyo (Pennisetum clandestinum Hochst Ex Chiov). Pastos y Forrajes 37(1), 31-37.

Miles, N., Thurtell, L., Riekert, S., 2000. Quality of Kikuyu herbage from pastures in the Eastern Cape coastal belt of South Africa. South African Journal of Animal Science 30 (Supplement 1), 85-86.

Municipio de Duitama, 2014. Sistema de Información Oficial, Colombia. http://www.duitama-boyaca.gov.co/indicadores_anuales.shtml?apc=bexx-1-\&x=2998187

Muscolo, A., Panuccio, M.R., Eshel, A., 2013. Ecophysiology of Pennisetum clandestinum: a valuable salt tolerant grass. Environmental and Experimental Botany 92(1), 55-63.

Osorio, F., 1996. Efecto de la condición corporal sobre la producción y reproducción en ganado lechero. Avances tecnológicos de la producción lechera, Finca S.A, Colombia, pp. 21-26.

Ortega, S., Acevedo, C., 1999. Programación del riego en sistemas por surco y goteo, Comisión Nacional de Riego,
Universidad de Talca, Chile, pp. 17-20. http://www.citrautalca.cl/intranet/archivos/1164912291.pdf

Rautenbach, E., Van Ryssen, J.B.J., Van Niekerk, W.A., 2008. Changes in nutrient composition of kikuyu foggage as winter progressed. South African Journal of Animal Science 38, 342-349.

Reeves, M., Fulkerson, W.J., Kellaway, R.C., 1996. Forage quality of kikuyu (Pennisetum clandestinum): the effect of time of defoliation and nitrogen fertilizer application and in comparison with perennial ryegrass (Lolium perenne). Australian Journal of Agricultural Research 47(8), 1349-1359.

Read, J.W., Fulkerson, W.J., 2003. Managing kikuyu for milk production; Agfacts P 2.5.3, $3^{\text {th }}$ Ed. State of New South Wales, NSW Agriculture. pp. 2-4. https://www.google. cl/?gfe_rd=cr\&ei=zDtIVaCFKsrhwATbnIDoDw\&gws_rd $=\mathrm{ssl} \# \mathrm{q}=$ Managing + kikuyu+for + milk + production+Agfac $\mathrm{t}+\mathrm{P} 2.5 .3 \% 2 \mathrm{C}+$ third+edition $\% 2 \mathrm{C}+$ revised + March +2003 $+\mathrm{JW}+$ Read1+WJ+Fulkerson

Sbrissia, A., Euclides, V.P.B., Barbosa, R.A., Montagner, D.B., Padilha, D.A., Santos, G.T., Zanini, G.D., Duchini, P.G., Da Silva, S.C., 2013. Grazing management flexibility in pastures subjected to rotational stocking management: herbage production and chemical composition of kikuyugrass sward. Proceedings $22^{\text {nd }}$ International, Grassland Congress, Australia, pp. 1038-1040. http://www.internationalgrasslands.org/files/igc/publications/2013/ proceedings-22nd-igc.pdf

Sinclair, K., Lowe, K.F., Pembleton, K.G., 2007. Effect of defoliation interval and height on the growth and quality of Arachis pintoi cv. Amarillo. Tropical Grasslands 41(4), 260-268.

Soto, C., Valencia, A., Galvis, R.D., Correa, H.J., 2005. Efecto de la edad de corte y del nivel de fertilización nitrogenada sobre el valor energético y proteico del pasto kikuyo (Pennisetum clandestinum). Revista Colombiana de Ciencias Pecuarias 18(1), 17-26.

Vander Colf, J., Botha, P.R., Meeske, R., Truter, W.F., 2013. The production potential of Kikuyu (Pennisetum clandestinum) over-sown with ryegrass (Lolium spp.) in a no-till system. Proceedings of the $22^{\text {nd }}$ International Grassland Congress, Australia, pp. 476-478. http://www.internationalgrasslands.org/files/igc/publications/2013/proceedings-22nd-igc.pdf

Van Soest, P.J., Robertson, J.B., Lewis, B.A., 1991. Methods for dietary fiber, neutral detergent fiber and nonstarch polysaccharides in relation to animal nutrition. Journal of Dairy Science 74, 3583-3597.

Vela, J., Vargas, R., 2009. Caracterización de la dinámica de producción de materia seca del kikuyu (Pennisetum clandestinum) asociado con árboles y en pastoreo para producción de leche en el trópico colombiano. Revista Ciencia Animal 2, 27-40.

Whitney, A.S., 1974. Growth of kikuyu grass (Pennisetum clandestinum) under clipping. II. Regrowth characteristics in relation to nitrogen fertilization and climate. Agronomy Journal 66(6), 763-767.

Zhai, L., Feng, Q., Li, Q., Xu, C., 2010. Comparation and modification of equations for calculating evapotranspiration (ET) with data from Gansu province, Northwest China. Irrigation and Drainage 59, 477-490. 